\title{
La adaptación del fondo léxico patrimonial en la Historia general de las cosas de Nueva España
}

\author{
Teresa Bastardín Candón* \\ Universidad de Cádiz, España
}

\begin{abstract}
Resumen
A partir de la Historia General de las cosas de Nueva España, de fray Bernardino de Sahagún, analizamos una muestra representativa del proceso de adaptación léxica al español americano, con objeto de descubrir, por una parte, la incorporación de algunas de estas voces al habla del México colonial, por otra, con la intención de proporcionar noticias sobre los aspectos más relevantes que se observan en esta nominación, y de mostrar, en el caso de nuestro fraile, su fina capacidad de percepción de la realidad americana, acorde con su labor científica y con su grado de aclimatación a las nuevas tierras.

Palabras clave: español de América, léxico colonial, variedades léxicas.
\end{abstract}

Para correspondencia, dirigirse a: Teresa Bastardín Candón (teresa.bastardin@uca.es), Departamento de Filología, Facultad de Filosofía y Letras, Universidad de Cádiz, Avda. Gómez Ulla, 1, 11003 Cádiz, España. 


\title{
The AdJustment of THE PATRIMONIAL VOCABUlary IN THE Historia GENERAL DE LAS COSAS DE NUEVA ESPAÑA
}

\begin{abstract}
From the Historia General de las cosas de Nueva España, by fray Bernardino de Sahagún, we analyze a representative sample of the process of lexical adjustment to American Spanish, in order to discover, on the one hand, the incorporation of some from these voices to the speech of the colonial Mexico, on the other hand, with the intention of providing news on the most relevant aspects that are observed in this nomination, and of showing, in case of our friar, his thin capacity of perception of the American reality, according to his scientific work, and with his degree of acclimatization to the new lands.
\end{abstract}

Key words: Spanish of America, colonial lexicon, lexical varieties.

Recibido: 03/05/13 Aceptado: $14 / 05 / 13$

\section{INTRODUCCIÓN}

Como en todos los hombres de su tiempo, en fray Bernardino de Sahagún se observa el proceso de acriollamiento que sufre el español al adaptarse a la nueva realidad americana, con procedimientos no muy diferentes a los de otros cronistas, que confirman una experiencia cultural e idiomática común (Rivarola, 1990: 63). El proyecto etnológico y lingüístico de la Historia, pensada y escrita inicialmente en la lengua aborigen, requiere, no obstante, de un texto bilingüe que muestre las equivalencias léxicas en las dos lenguas, náhuatl y castellana ${ }^{1}$. Este método, con cierto grado de tradición en la labor indigenista franciscana, dista mucho de representar los usos reales de la lengua española en América² $^{2}$ y solo ofrece ejemplos

1 Manejamos la edición facsímil de la Historia general de las cosas de Nueva España (Sahagún 1979). Existe un ejemplar en la Escuela de Estudios Hispanoamericanos de Sevilla y otro en la Academia de la Historia, en Madrid.

2 Los ejemplos abundan, particularmente, en la descripción de la flora y fauna americanas, con el registro de términos cuya vitalidad debió de ser escasa en el habla de los conquistadores, 
comparativos en el contexto general del conocimiento propio del humanismo o representaciones conceptuales aproximadas, especialmente en el más específico de la evangelización ${ }^{3}$. La certera visión lingüística de nuestro franciscano, con comentarios que prueban la vigencia de las voces, e, incluso, con testimonios de variantes a uno y otro lado del Atlántico, nos permitirá seguir y valorar la incorporación del americanismo léxico y semántico. Su introducción en el texto castellano, con plena vitalidad y uso, confirma el grado de aclimatación léxica en la lengua de nuestro franciscano y asimismo su extensión en el habla del México colonial, con la adaptación de no pocos términos patrimoniales como primer recurso de nominación de la naturaleza americana.

\section{LA INSUFICIENCIA LÉXICA Y REFERENCIAL}

Este proceso de adaptación del fondo léxico patrimonial representa el primer esfuerzo de captación de la nueva realidad bajo los parámetros de lo conocido. La búsqueda de referentes peninsulares, que ayudaran a la descripción de la naturaleza y los modos de vida indígenas, es una constante desde los primeros tiempos de la conquista que ilustra sobre la necesidad de hallar una base comparativa capaz de representar y fijar conceptualmente lo que hasta entonces carecía de imagen en la mente de los nuevos pobladores.

Desde muy pronto, la dificultad de esta empresa se hace patente. Sobradamente conocida es la desazón que produce en el Almirante la falta de referentes peninsulares que sirvieran de base comparativa para el nuevo mundo que trata de describir; o la indecisión mostrada al dar nombre a la nueva naturaleza. Numerosos son, asimismo, los testimonios cronísticos que nos hablan del interés por precisar el contenido referencial de aquellos vocablos de nuestra propia lengua que habían adquirido matices

a menudo por lo extraño del referente. Los propios comentarios del franciscano reflejan esta situación, cuando refiere el contexto geográfico de las voces empleadas, o el olvido del vocablo patrimonial: "ay vnos lagartillos del agua [...]. EStos se crían también en los lugares húmedos, pienso es vaqueruela de Castilla (L. XI, f. 228r.); "Vnos gusanos [...] que también los ay en España. Llámanse coiaiaoal, en la lengua española no sé como se llaman (L. XI, f . 249v.).

Si fray Bernardino actúa en este sentido como un científico que registra en su obra todo el saber enciclopédico de la cultura azteca, no hay que olvidar que su interés lingüístico se centra en la lengua conquistada, como instrumento de predicación y catequización. 
o significados nuevos en el proceso de adaptación al mundo americano ${ }^{4}$. El propio fray Bernardino de Sahagún se hace eco de esta insuficiencia léxica y referencial en varios pasajes de su obra. El franciscano comparte con los nuevos pobladores la necesidad de nominación y de descripción de las tierras descubiertas, con aprovechamiento del bagaje léxico peninsular, y con referencias que recuerdan su lugar de procedencia ${ }^{5}$, "ay en eSta tierra vnos gusanos que en CaStilla la Vieja se llaman carralexas, se crían en las vinas, llámanlas tlalxiqujpilli” (L. XI, f. 248v.); "a la yerua que comen los cauallos en eSta ciudad de México llaman caltolli, házeSe en el agua, eS triangulada. En algunas partes de CaStilla Se llama carrizo" (L. XI, f. 335r.) ${ }^{6}$; su celo científico y su fina capacidad de observación se muestra, además, en la búsqueda constante de esa base comparativa que era la realidad peninsular, "Este anjmal que se llama quanmjztli por las propriedades paresçe ser onza y sino lo es no se a que otro anjmal sea semejante" (L. XI, f. 160v.); "eSte anjmal, por la relación, paresce que es oso, y si no es oSo no sé a que anjmal se compare de los que conoscemos" (L. XI, f. 160r.) / tx. n. cuitlachtli; y en la preocupación por la propiedad lingüística de las

4 Algunas citas sobre este aspecto lingüístico del descubrimiento americano pueden verse, entre otros, en Morínigo (1959: 233-241), Alvar (1976: 45), o Enguita Utrilla (1991: 201).

5 Sobre la presencia de la Tierra de Campos en la obra de nuestro franciscano, con otros ejemplos, véase Frago (1995). Los vocablos propios de su modalidad dialectal, que afloran en la Historia, y su posible extensión y adaptación a la variedad americana la hemos tratado en Bastardín Candón (2011), asimismo, con referencia a otros términos de adscripción regional. Otras referencias son más generales, como "Vsauan de atambor y de tamboril, el atambor era alto, como hasta la cinta, de la manera de los de España en la cubertura" (L. VIII, f. 267v.); "También tiene por oficio [...] hacer mantas de tela rala, como es la toca, y por el contrario hacerlas gruesas de hilo gordazo o grueso, a manera de cotonía de Castilla" (L. X, f. 38v.); "Ay vna aue en eSta tierra que se llama quetzaltototl [...] es tan grande como vna ave que se llama Tzanatl que es tamaña como vna vrraca o pega de España" (L. XI, f. 173v.); "A las avaneras de los ríos llaman atzcalli. Véndenlas y cómenlas, tienen la concha negra como las de España que se hazen en los ríos" (L. XI, f. 216v.); "Vnos coqujllos del agua que llaman axaxaiacatl o quatecomatl. Son por la mayor parte negros y del tamaño de pulgón de Castilla y de aquella hechura" (L. XI, f. 220v.), por citar algunas.

${ }^{6}$ Otros contextos son más generales, pero muestran, asimismo, esta búsqueda referencial: "Vsauan de atambor y de tamboril, el atambor era alto, como hasta la cinta, de la manera de los de España en la cubertura" (L. VIII, f. 267v.); "También tiene por oficio [...] hacer mantas de tela rala, como es la toca, y por el contrario hacerlas gruesas de hilo gordazo o grueso, a manera de cotonía de Castilla" (L. X, f. 38v.); "Ay vna aue en eSta tierra que se llama quetzaltototl [...] es tan grande como vna ave que se llama Tzanatl que es tamaña como vna vrraca o pega de España” (L. XI, f. 173v.); “A las avaneras de los ríos llaman atzcalli. Véndenlas y cómenlas, tienen la concha negra como las de España que se hazen en los ríos" (L. XI, f. 216v.); "Vnos coqujllos del agua que llaman axaxaiacatl o quatecomatl. Son por la mayor parte negros y del tamaño de pulgón de Castilla y de aquella hechura" (L. XI, f. 220v.), por citar algunas. 
nuevas denominaciones, "ay en esta tierra vn anjmal que se llama coiotl, al qual, algunos de los espanoles, le llaman zorro y otros le llaman lobo, y según Sus propriedades, a mj ver, nj es lobo nj zorro, sino anjmal proprio desta tierra" (L. XI, f. 161v.).

\section{LA ADAPTACIÓN LÉXICA}

A pesar de todo, la necesidad de dotar de un nombre a las nuevas especies de fauna y flora, a los instrumentos y objetos relacionados con el quehacer diario, a la vestimenta o la alimentación, y a los aspectos relativos a la organización social de los distintos pueblos encuentra en el recurso comparativo y en la recurrencia a las voces de la propia lengua, con clara inclinación hacia los términos de procedencia árabe en los primeros momentos (Morínigo, 1964: 234-235), el mecanismo de nominación y asimilación más efectivo, antes de que el contacto con los pueblos aborígenes permitiera la adquisición del préstamo léxico, como solución más general ante la incapacidad designativa del vocabulario patrimonial ${ }^{7}$.

Así se explican las continuas connotaciones de los términos europeos que se registran en la obra de nuestro fraile, no ajenas a otros $\operatorname{cronistas}^{8}$, que se aplicaron como designación de la naturaleza y los modos de vida indígenas, o la adaptación y generalización de otras voces que exigía la acomodación al mundo colonial. Se encuentran en las páginas de la Historia multitud de

\footnotetext{
El proceso, iniciado en las islas, resulta un mecanismo constante al que los conquistadores recurren ante cada territorio descubierto, con unos resultados que reflejan el peso del bagaje peninsular que los motiva y condiciona, pues su carácter espontáneo no impide la inevitable sujeción que la tradición lingüística y cultural impone al acto creativo. Esto explica la diversidad de nombres que, aplicado a una misma realidad, se descubren en todo el continente americano, y la identidad nominativa de tantas especies distintas. Ni siquiera los términos patrimoniales de carácter general obligan a considerar una primitiva génesis insular, y, posiblemente, haya que considerarlos como resultados paralelos que se desarrollan en áreas geográficas distintas (Enguita Utrilla, 1987: 143).

8 Desde los trabajos de Alvar (1970 / 1990, 1972, 1976, 1987, 1992), han sido muchos los estudios que analizan obras americanas bajo esta perspectiva; así los de Enguita Utrilla (1979a, 1987), con datos de Fernández de Oviedo; Rivarola (1990), sobre las obras de Pedro Pizarro y Pedro Cieza de León; Franco Figueroa (1991a), sobre documentación indiana; Frago (Frago y Franco Figueroa, 2003), con datos de Guamán Poma de Ayala y de José Acosta. Contamos, además, con el estudio de conjunto del profesor Boyd-Bowman (1972).
} 
estos nombres empleados en la denominación de los especímenes de plantas y animales, aunque no todos representen un uso y vitalidad contrastados.

Las distintas voces con las que se nombraron los cérvidos americanos, como adive, ciervo o venado, con referencia, fundamentalmente, al mazate (n. mazatl), "andauan los demás teuchichimecas veStidos de otros pelleios de venado o de adiues" (L. X, f. 124r.), "ay cieruos en esta tierra de muchas maneras, viven en las montañas, son altos de cuerpo, tienen las piernas largas" (L. XI, f. 169v.) / tx. n. maçatl; o las de los felinos, como león, tigre, onza, gato cerval o gato montés, para el ocelote (n. ocelotl) y otras especies indígenas, "si acertauan a matar algún león o tigre, gato montés, conejos o venados le preSentauan el pelleio y la carne" (L. X, f. 123r.), "tenjan cargo de guardar tigres y leones y onças y gatos ceruales" (L. VIII, f. 280v.), reflejan, una vez más, la vacilación en esta adaptación léxica, que ya vimos en la nominación del coyote (n. coyotl) y otros mamíferos emparentados, también con los nombres de lobo, zorro, o raposo, "el lobo o çorro no trae conSigo el fuego para cozer o asar lo que a de comer" (L. VI, f. 193v.); "ay otro anjmal desta especie al qual llaman tlalcoiotl [...], a eSte llámanle algunos zorro o raposo" (L. XI, f. 163r.).

No faltaron otras denominaciones generales como avión, nombre de un tipo de golondrina mexicana y de otras clases de aves americanas, "ay aviones en esta tierra como los de Castilla y crían como los de Castilla en sus casitas de tierra" (L. XI, f. 181v.) / tx. n. acuicuialotl; comadreja, marsupial americano que fray Bernardino identifica con el coçatli, "la comadreja es delgadilla, tiene la colilla largujlla, tiene la cara manchada, es bermejuela, tie el pecho blanco" (L. XI, f. 168r.) / tx. n. coçama o coçatli; cuervo, como designación del cacalote (n. cacalotl) o cuervo común de México, "ay también cueruos como los de España, llámanlos cacalutl o calli o cacalli" (L. XI, f. 198v.); escarabajo, para el pinacate (n. pinacatl), "ay también escorabajos como los de España y llámanlos pinacatl, son negros y hieden como los de España" (L. XI, f. 250v.) ${ }^{9}$; o gavilán, que nombra a distintas aves rapaces americanas, y en la crónica de nuestro fraile, al itztlotli mexicano, "ay también cavillanes como los de España, de la mjsma color y del mjsmo tamaño y de las mjsmas costumbres" (L. XI, f. 200v.) / tx. n. iztlhotli. En el ámbito de la flora, abundan, igualmente, estas designaciones. El término ciruela fue la denominación más común del mazajocote (n. mazaxocotl), "A los árboles en que se hazen las ciruelas llaman maçaxocotl. Házense en tierras

\footnotetext{
9 Y otras especies: "ay también ay eScarabajos como los de CaStilla que hazen pelotillas del estiércol y llévanlo rodando, llámanlos tecujtlaololo» (L. XI, f. 250v.).
} 
calientes, el fruto deStos árboles, vnos son coloradas, otros amarillas, vnas grueSsas, otras menudas" (L. XI, f. 274v. $)^{10}$; la voz doradilla designó a una especie de helecho llamado en la lengua tequequetzal, "ay doradilla en eSta tierra y llámanla tequequetzal" (L. XI, f. 336r.). Algunas de las variedades de árboles americanos más conocidas recibieron los nombres de ciprés o cedro, como el ahuehuete (n. ahuehuetl), según reconoce Santamaría en su diccionario de mexicanismos, "atapados con hojas de cedro que llaman aueuetl" (L. II, f. 137r.); "vende todo género de leña, ciprés, cedro, pino" (L. $\mathrm{X}, \mathrm{f}$. 61r.) / tx. n. aueuetl, incluso con noticias sobre el uso de estas voces, "ay en eSta tierra vnos árboles muy grandes y dízense cedros, tienen la hoia muy menuda, tienen agallas como de los cipreSes, aunque más pequeñas" (L. XI, f. 264v.) / tx. avevetl; pino o abeto fueron los nombres más usados para el ocote (n. ocotl) y el oyamel (n. oyametl), "ay piños en eSta tierra como los de España, házense en ellos piños y piñones" (L. XI, f. 264r.) / tx. n. ocotl, también con referencias sobre la generalización de los vocablos y de sus referentes, "ay otros árboles en eSta tierra que se llama oiametl, no ay en España árboles de eSta manera (que yo sepa de eStos), se coge vn licor muy precioso, muy medicinal que se llama abeto, no le vsavan los yndios, nj le conocían, agora en eStos tiempos se a hallado" (L. XI, f. 264r.); y el aguacagüite (n. ahuaquahuitl) o encina común se llamó roble, "ay también en eSta tierra robles que se llaman avaquavitl" (L. XI, f. 265r.) ${ }^{11}$; "vende la leña de roble y de pino y de fresno y de madroños y la leña que respenda y humea mucho" (L. X, f. 61v.) / tx. n. aoatl. El mismo reconocimiento del uso real de estas denominaciones se encuentran para la voz peruétano, con referencia al chicozapote (n. xicotzapotl), "ay otros árboles que se llaman xicotzaputl, llámanlos los españoles peruétanos” (L. XI, f. 273v.), que todavía se recoge en algunos de los diccionarios de americanismos, como el de Santamaría, para México, o el general de Neves; y para el término cerezo, como denominación del capulín (n. capolin), aunque el fruto vaya acompañado del sintagma preposicional, "Vnos árboles [...] que se llaman capuli o capulquavitl y a los eSpañoles llaman a eStos cerezos, porque son

10 Las distintas clases recibieron, asimismo, esta denominación, con el adjetivo de color: "otra manera de fruta se llama maçaxocotl chichiltic, vna manera de ciruelas y son coloradas; cuztic maçaxocotl, otra manera de ciruelas y son amarillas; tlaztaleoaltic maçaxocotl, otra manera de ciruelas y son bermejas o naranjadas" (L. VIII, f. 274r.).

11 También para otros tipos de robles: "ay vnos robles en eSta tierra, la corteça es grueSsa como vn dedo o como dos dedos, vsSan della para teñjr y para cordir los cueros” (L. XI, f. $265 \mathrm{v}$.) / tx. n. quauhtepuztli; "ay también vnos robles o carrascos muy rezios de que hazen coas que los llaman vicquavitl, naçen en las peñas y en los riscos” (L. XI, f. 265v.). 
algo semejantes a los cerezos de España en la hoia y en el fruto. La fruta se llama capuli, qujere dezir cerezas deSta tierra" (L. XI, f. 276v.).

En todas estas denominaciones, el alto grado de similitud entre los referentes permitía esta transferencia léxica ${ }^{12}$, según constata el franciscano, aunque, en ocasiones pondere la calidad de las especies indígenas, como ya hiciera el propio Colón, ante la enormidad de la naturaleza americana, "alcon, açor, cavillán. Ay estas aues arriba dichas en nueStra tierra y aún dizen los españoles que Son mejores que las de España" (L. XI, f. 200v.); o advierta de la desemejanza entre tantas especies, como sucede con el camarón mexicano o chacalín (n. chacallin), diferente en forma y tamaño, "los camarones buenos críanSe en la mar y en ríos grandes, y en manantiales de los ríos, son mayores que los camarones de por acá, son colorados y muy sabroSos" (L. XI, f. 215v.) / tx. n. chacali; con el gorrión, designación del pájaro denominado en la lengua molotl, "ay gorriones en esta tierra, pero difieren de los de España porque son algo menores, aunque también traujejos como los otros" (L. XI, f. 203v.); con el moral o amacapulin (n. amacapolin o amacapolquahuitl), cuyo fruto es más pequeño que el de las especies

12 El mecanismo de comparación en la captación de la nueva realidad arroja abundantes testimonios sobre los procedimientos analógicos más recurrentes que sirven de base en la nueva nominación. La similitud de formas, abundante en la descripción de la flora y fauna americanas, se aprecia en el establecimiento de equivalencias entre unos y otros referentes con procedimientos sintácticos explícitos: "ciertas sauandijas a manera de aradores" (L. X, f. 108r.); " cierta yerua áspera llamada çacamalinalli que es a manera de espartillo" (L. X, f. 102r.); en ocasiones, avaladas por un uso derivativo que incide en la variación dimensional, como verdejoncillo, "ay otra auecita que se llama mjaoatototl o xopan tototl, es como verdejoncillo [...], es pequeñjto" (L. XI, f. 208r.), para designar a este tipo de ave, basada en la formación analógica, a partir de verderón; o perejón, "ay otra yerua medicinal que Se llama oqujchpatli [...], quando Se les cayen las flores hace vna frutilla como perejones" (L. XI, f. $327 \mathrm{r}$.), con que se designa a una especie de fruta parecida a una pera grande. No obstante, las referencias se concentran en la semejanza parcial de las especies que se relacionan, como en el caso de plantas y flores, parecidos en hojas, ramas o frutos; "ay otra manera de árboles que se llaman ocotzoquavitl o xochiocotzoquavitl [...] tienen las hoias como alisos" (L. XI, f. 267v.); "la yerua de que hezimos arriba mención que Se llama xiloxochitlacotl es mata, tiene la rramas maciças y delgadas y redondas como los pinpollos del membrillo" (L. XI, f. 312v.); "ay vnos árboles que se llaman vaxi o vaxquavitl [...] crían vna fruta como algarrovas, es de comer" (L. $\mathrm{XI}, \mathrm{f} .275 \mathrm{v}$.); "ay en eSta tierra palmas naturales [...] su fruta es dulce y es de comer, es como dátiles" (L. XI, f. 266v.); o en el de la fauna, con recurrencia a características como el color o el tamaño: "ay también cernjcalos como los de España, y la color dellos es como la color de los de España" (L. XI, f. 200r.); "ay otra aue del agua que se llama achalalactli [...] es del tamaño de vna cerceta" (L. XI, f. 193v.); "ay vn árbol medicinal que Se llama yohoalxochitl, es grande como vna yguera" (L. XI, f. 320r.). Hay que recordar, además, que la mayoría de las voces que aquí se registran se encuentran en los diccionarios de americanismos, como designación de distintas variedades autóctonas. 
europeas, "ay morales en eSta tierra, llámanlos amacapuli o amacapulquavitl, es liso y acopado, tienen muchas ramas y hoias, y las hoias son muy verdes, son algo vellosas las hoias por el envés, llevan moras como las de CaStilla, pero pequeñuelas" (L. XI, f. 276v.); o con el topo, que fue la denominación más general de la tuza (n. toçan), y la única que registra el franciscano, que no recoge el término en la lengua náhuatl, "los topos deSta tierra son grandes, como grandes ratas, tienen el pelo bermejo, son carnudos, y gordas, de los pies bajos, casi rrastra la barriga" (L. XI, f. 171v. $)^{13}$.

Las condiciones del terreno propician, asimismo, la expansión y consolidación histórica de un vocabulario de menor empleo y extensión peninsular. Se trata de voces como cerro, "dexaron también vna sierra o vn cerro que los dichos tultecas començaron a hazer y no lo acabaron" (L. X, f. 116v.); "en el cerro o monte de Cholollan" (L. X, f. 145r.); monte "terreno cubierto de arbustos', "el que vende escouas valas a segar en el monte con hozes" (L. XI, f. 65v.); barranca 'mole de tierra o piedra sobre una quiebra o corriente de agua', "hablo con vna persona de gran mageStad en cuya preSencia corre vn río que tiene vna barranca profundíSsima y preziSsa o taiada" (L. VI, f. 10v.); "tú mjsmo te arrojaste en la barranca del río y caýste en los laços y redes" (L. VI, f. 26v.), con su derivado americano desbarrancar, "¿qué haré Si desbarrancare o despeñare por mj culpa a los que tengo de regir" (L. VI, f. 38v.); y de otras, con una adscripción diatópica más precisa, de ascendencia occidental, como ciénaga, "ay otras ranas que llaman çoqujcujatl, que qujere dezir ranas de cieno, y críanse en las ciénagas" (L. XI, f. 219v.); "ay otra yerva que se llama atlepatli, críase en las orillas del agua y cerca de las ciénagas, es mortal" (L. XI, f. 283r.); y páramo, "los mexicanos o naoas y todos los otros prosigujeron su camjno por los llanos o páramos para descubrir tierras" (L. X, f. 148r.); "pones a rriesgo tu vida y Salud, atreujéndote, sin temor, a subir y descendir riscos y barrancas y montes y páramos, con fatigas y trabajos" (L. IX, f. 348r.) ${ }^{14}$.

En el ámbito de la alimentación, la crónica sahaguntina constata la adaptación de voces que gozan de un reconocimiento histórico y actual en el territorio americano, como tortilla, "comales que Son tortas de barro cozido para cozer las tortillas en ellas" (L. X, f. 62r.), con referencia al tamal (n. tamalli), según la descripción que proporciona el franciscano, "tamales que son como vnos pastelejos redondos hechos de mahíz" (L. I., f. 33v.); "tamales, son como panes redondos, hechos de mahíz, nj bien

\footnotetext{
Todavía Santamaría recoge topo, como denominación de la tuza o taltuza.

Véanse Frago, 1999: 45; Franco Figueroa, 2001: 530, Bastardín Candón, 2011: 20,
} 22). 
rollizos nj bien redondos" (L. I, f. 24v.), y de los contextos en los que se coordinan ambas voces, "echa tortillas o tamales" (L. XI, f. 289r.), aunque, en ocasiones, emplee el término empanada, como 'empanadilla', para referirse al nacatamal (n. nacatamalli) o tamal relleno de carne, "de allí Se traen las buenas enpanadas de gallinas, nacatamalli" (L. X, f. 136r.) $)^{15}$. Asimismo, la generalización y vitalidad de voces de origen dialectal se muestra en frijol, "eSta yerua haze vnas avas que son como los frisoles grandes y son frisoles SylueStres" (L. XI, f. 285r.), de amplio uso americano, y ascendencia occidental ${ }^{16}$.

La descripción de los distintos grupos indígenas es objeto, asimismo, de un proceso nominativo en el que no faltan algunas de estas acomodaciones léxicas. Representativo de la indumentaria es el término manta 'tela de algodón usada como prenda de vestir', "su officio es tratar en las mantas de los hombres y en las camjSas de las mugeres desta tierra" (L. X, f. 47r.); "y las que vende, aora Sean mantas, aora sean naguas y vipiles son ya traýdas y viejas" (L. X, f. 47v.), y la peculiar fisonomía india está representada con términos como crenchado, "cortáuanle los cabellos como a cuextecatl, desiguales y malcortados y espelucados y crenchados" (L. IX, f. 362v.), que adquiere, en algunas partes de América, el sentido más específico de 'cabellos despeinados', según $E A$, y es el sentido aproximado que se infiere del contexto sahaguntino.

La organización social de los pueblos amerindios, con sus jefes y caciques, favoreció el sentido más restringido de voces como mandón, "entre ellos auja Señores y mandones que mandauan a Sus Súbditos" (L. X, f. 127v.); principal, "todos los quales biujan en policía y tenjan Sus repúblicas Señores, caciques y principales" (L. X, f. 126v.); "Motecuçoma juntó algunos adivjnos y agureros y algunos principalejos y los embió al puerto donde eStauan los españoles" (L. XII, f. 419r.); o cuadrillero, "desque le aujan llegado al patio del cu, luego començauan los tlaiacanques o quadrilleros y dauan bozes muy fuertemente para que se juntasen todo el pueblo" (L. II, f. $115 \mathrm{v}$.$) . Las actividades comerciales, tan frecuentes entre los indios, explica$ el uso y pervivencia de rescatar 'comerciar', 'revender', "el que rescata

15 La alternancia de ambas voces, empanada y empanadilla, se constata en las explicaciones que proporciona el franciscano: "hechar maSa de frisoles cozidos en la masa de los tamales y hazer tamales de carne como enpanadillas y hazer bollos redondos" (L. X, f. 39v.). Moliner, s. v. empanada, registra en su catálogo de referencia las voces tamal y nacatamal.

16 Véanse Frago 1999: 18, 19, 57; Franco Figueroa 2001: 530; Bastardín Candón 2011: 22). 
con plata es mercader" (L. X, f. 46r.), que se atestigua desde los primeros cronistas, y reconocen Autoridades y Terreros como voz usada en Indias ${ }^{17}$, y su derivado rescatador, "el rescatador regatón suele engañar en lo que vende" (L. X, f. 46v.), también con un temprano reconocimiento académico, igualmente, en Autoridades. La voz regatón 'vendedor al por menor', "el mercader suele Ser regatón y Sabe ganar y prestar a logro, concertarse con los comprantes y multiplicar la hazienda" (L. X, f. 31r.), y su variante recatón, por confusión con rescatar, "el que es recatón en eSte officio es muy carero y encarecedor de las cotaras que vende" (L. X, f. 55v.), tienen un uso mexicano más específico, según Santamaría y Morínigo, como 'vendedor en plazas y mercados', y con indicación sociolingüística, como voz usada en la capital para nombrar a los revendedores de Xochimilco.

\section{DERIVACIÓN Y COMPOSICIÓN}

Igualmente productivo en este proceso de nominación fue el mecanismo lexicogenésico de la derivación, con ejemplos que abarcan todos los ámbitos referidos. La voz pipiana 'guiso indiano', recogida ya por Autoridades y Terreros, en su variante más extendida, pipián o pepián, cuenta, además, con el testimonio sahaguntino, que verifica su empleo generalizado, "también comjan los Señores muchas maneras de caçuelas, vnas dellas se llaman totolin patzcalmollo, q. d., caçuela de gallina hecha a su modo, con chilli bermejo y con tomates y pepitas de calabaças molidas, que se llama agora pipiana" (L. VIII, f. 273v.). El vocabulario relativo a los adornos está presente en términos como bezote, con una importante carga simbólica en la cultura india, como recuerda Sahagún, "los que dellos eran Señalados por valientes y que podían traer beçotes" (L. II, f. 106r.); "lleuauan vn beçote hecho de pluma" (L. II, f. 117r.); barbote; u orejera, "buscauan que eStos eSclauos fuesen muy regalados y muy bien ataujados, con aquellos adereços que ellos vsauan de orejeras y barbotes" (L. I, f.13r.); la voz chorchero, con escasa documentación histórica, y uso en México, fue ya el calificativo aplicado

17 En México es 'comerciar con los índígenas', según el DCECH, s. v. regatear; SM, Morínigo, Neves y Malaret recogen el sentido de 'traficar de pueblo en pueblo', en Colombia y otras partes de América del Sur. El diccionario de americanismos de Santamaría imputa su uso a América Meridional, y como 'revender', a México. 
a determinadas tribus, por su carácter fiestero, "Son pinoles y chontales y nonovales, Son grandes chorcheros" (L. XI, f. 381v.) ${ }^{18}$; y la variante parcho, "se pondrá vn parcho para acabar de sanar" (L. X, f. 115r.), referida en el diccionario académico, $\mathrm{y}$ en los generales de americanismos, a la zona caribeña, revela un uso histórico más extenso, y en la crónica sahaguntina, es recurrente en la descripción de las prácticas curativas indias ${ }^{19}$.

No solo en el terreno de la vieja cultura india, también las actividades mineras y comerciales que se establecen en la colonia traen aparejadas el uso de un vocabulario más específico, como barreta 'barra de metal', "y el oro fundiéronlo y hizieron barretas" (L. XII, f. 434v.); "aquj también tomaron mucho oro en barretas, y embasijas y oro en poluo" (L. XII, f. 151v.); o tomín 'moneda de plata que se usaba en algunas partes de América', "nj entre los seglares nj entre los ecclesiásticos no ay nadie qujen los fauorezca nj con Solo vn tomjn" (L. X, f. 85v.); "ay vnas yerbazuelas en el agua que tienen la hoja como tomjn, anchuela, eStendida Sobre el agua" (L. XI, f. 335v.).

Estas derivaciones, con preferencia por los morfemas diminutivos, fueron, fundamentalmente, recurrentes en la designación de las variedades autóctonas de la flora y de la fauna americanas, con ejemplos como perrillo, probable denominación del 'perro de Chihuahua', también presente en otros autores de la época, que, de manera análoga, destacan su importancia para el abastecimiento cotidiano, atraídos por la perplejidad de esta práctica india, "también se proueýa de perrillos para comer" (IX, f. 345v.); "comprauan gallinas y perrillos para comer" (L. X, f. 130v.). Los hábitos alimentarios de los distintos pueblos se convertían de este modo en un índice de su grado de civilización y de cultura, como recuerda Sahagún, al hablar del pueblo otomí, que comía, entre otras cosas, zorrillos, "eStos otomjtes comjan los zorrillos que hieden y culebras y lirones y todo género de ratones y las comadrejas y otras Sauandijas del campo y monte y lagartijas de todas Suertes y abejones y langoStas de todas maneras" (L. X, f. 131r.), una denominación que todavía hoy mantiene su vigencia en el ámbito americano, con el sentido de 'mofeta' (n. epatl) ${ }^{20}$.

18 La voz no se recoge en los bancos de datos académicos, ni se registra en los diccionarios generales, hasta la edición actual del académico. Sí lo traen $S M$, Morínigo y Neves, con imputación a México.

19 La edición actual del diccionario académico, la localiza en Puerto Rico; Morínigo, en Colombia y Venezuela; y Neves en las Antillas y Venezuela.

20 Según se recoge en el diccionario académico general, con referencia a El Salvador, Guatemala, Hondura, México, Nicaragua, y Uruguay, y en el de americanismos, incluso con sentidos figurados como el de 'vehículo policial provisto de dispositivos para lanzar gases', con empleo popular en Chile. 
La constatación del uso histórico de algunos de estos vocablos la proporciona el propio Sahagún, con sus comentarios lingüísticos, a propósito de la voz tortolilla, "ay vnas auecillas en eSta tierra que se llaman cocotli y a todos los españoles las llaman tortolillas, no Son tan grandes como las de Castilla" (L. XI, f. 204v.), que certifica su empleo generalizado, y justifica el uso del diminutivo, porque son de menor tamaño; o el término, que ya recoge Covarrubias con referencia a las Indias, cochinilla (s. v. grana), "en cierto género de tunas Se crían vnos gusanos que que llaman cuchinjllas apegadas a las hojas y aquellos gusanos tienen vnos Sangre muy colorada. ESta es la grana fina" (L. XI, f. 368v.), también con mención de la sustancia colorante que se obtiene de ella, la grana cochinilla, según se comprueba en la identificación léxica que establece el nombre en la lengua. Con otro tipo de sufijo, también muestra este grado de asimilación lingüística la voz garzota, como denominación del aztate (n. aztatl) o garza indígena, con noticias sobre el proceso de dialectalización léxica del español en América, "ay vnas aves blancas que se llaman aztatl o teuaztatl, en algunas partes en España se llaman dorales, y acá las llaman garçotas blancas los españoles" (L. XI, f. 182r. $)^{21}$.

No faltaron tampoco los compuestos de palabras, abundantes, asimismo, en los nombres de plantas y animales, y en el de las sustancias, con voces como liquidámbar, "no Se da allí cacao nj el veinacaztli, Sino liqujdambar o la reSina oloroSa que llaman suchiocotzotl" (L. X, f. 135r.), con referencia al bálsamo o al árbol que lo produce, el ocozol; ni los efectos metonímicos, en variantes históricas que mantienen un uso regional americano, como se comprueba en la voz armado, frente al derivado armadillo, "del anjmal que llaman el armado" (S. L. XI, f. 153r.), que aún hoy pervive en Guatemala, Honduras, El Salvador y Costa Rica, según los datos del Diccionario de Americanismos académico $(D A A)^{22}$.

21 El empleo recurrente de la voz se aprecia en los distintos contextos seleccionados: "estos cabellos [...] atáuanlos con vnos hilos colorados a vnos penachos de garçotas, dos o tres" (L. II, f. 117v.); "dizen que este aue es el principio de las garzotas blancas que se juntan a él donde qujera que le veen" (L. XI, f. 175r.); "labrauan plumajes para baylar de plumas blancas y negras, de gallinas y de garçotas y de ánades" (L. IX, f. 369r.).

22 En otras ocasiones la aparición de algunas de estas voces en los diccionarios de americanismos corresponde a un registro libresco, que se perpetúa en la práctica lexicográfica, sin más apoyo documental que su aparición en obras literarias o históricas, como sucede con el término ciervoleón, que recoge Sahagún, más como traducción de la voz náhuatl maçamiztli, de maçatl 'venado' y miztli 'león', que como un uso real en la lengua de los colonos, “Ay vn anjmal en eStas partes que se llama maçamjztli, qujere dezir cieruo león, el qual no sé si le ay en njnguna otra parte, es del tamaño del cieruo y tiene la color de cieruo y tiene sus vnas como cieruo y los machos tiene cuernos como cieruo, pero tiene pescuños como león muy 


\section{LAS AGRUPACIONES SINTAGMÁTICAS}

Las agrupaciones sintagmáticas, que afloraron con la intención de aclarar la posible ambigüedad que acarreaba el empleo de voces castellanas en la nominación de la realidad indígena, presentan, en su mayoría, el denominador común que comprendía todas esas variedades y que coexistían en el sintagma de la tierra. Los ejemplos son abundantes, y algunos de ellos se repiten en otras obras cronísticas, aunque no todos presupongan un testimonio del empleo habitual de estas construcciones, y sí un recurso de aclaración conceptual ante la diversidad americana. En Sahagún, además, su asunción de lo americano favorece la aparición de variantes que muestran el grado de aclimatación a las nuevas tierras. De esta manera, el sintagma de esta tierra acompaña la descripción de las cerezas indígenas, los capulines americanos, como ya viera Alvar (1970 / 1990: 27), con datos de Bernal Díaz del Castillo, "La fruta se llama capuli, qujere dezir cerezas deSta tierra" (L. XI, f. 276v.). Asimismo, son de esta tierra o de la tierra las acederas, nombre con el que se identificó el jocoyol mexicano (n. xoxocoyolli), y que queda registrado en el diccionario de Terreros, aunque con la especificación de Indias, "ay otra yerua que se llama xoxocoioli y son las azederas desta tierra" (L. XI, f. 289v.); o el ajenjo, como denominación del estafiate (n. iztauhyatl), planta de gran uso medicinal, llamada así, como reconoce Sahagún, por su parecido con la castellana, aunque su especificidad es pareja a la vitalidad con la que debió emplearse el término, según la insistencia del franciscano en proporcionar la denominación castellana, frente a los significantes de la otra lengua, "yztac xoxocoioli, q.d. yerua azeda blanca o azederas desta tierra" (L. VIII, f. 274v.); "ay otra yerua que se llama xoxocoioli y son las azederas desta tierra" (L. XI, f. 289v.); "las yeruas comestibles como son las hojas de las matas de Chile, bledos, azederas, mastuerço, poleo" (L. X, f. 70v.). Asimismo, el ayote (n. ayotl) fue la calabaza de la tierra, "calabaças deStas tierras que Se comen" / tx. n. ayotli (L. XI, f. 404r.); el huisquelite (n. huitzquilitl), el cardo de la tierra, "ay otra yerua que se llama vitzqujlitl. Son cardos de la tierra, tienen espinas, y las hojas de abaxo Son cenjcientes y los de arriba Son verdes" (L. XI, f. 288v.); el izote (n. icçotl), la palma de la tierra, "mantas que se hazen de palmas que se llaman icçotl, de la tierra"

agudos y los dientes y culmjllos como león" (L. XI, f. 160v.), pero que aparece en $S M$, y en Neves, con base en la documentación sahaguntina. 
(L. X, f. 57r. $)^{23}$, aunque la misma denominación se aplicó a otros tipos de palmera indígena, como el zoyate (n. çoyatl), "ay en eSta tierra palmas naturales que Son como las de España, llámanlas çoyatl" (L. XI, f. 266v.); y el fruto del tejocote (n. texocotl o texocoquahuitl), la manzanilla de la tierra, "los árboles en que se hazen las mançanjllas de la tierra se llaman texocotl o texocoquavitl" (L. XI, f. 274r.) ${ }^{24}$; y todavía hoy son el nombre, ya sin la referencia geográfica, de distintas especies americanas de plantas y frutos, como sucede, asimismo, con el jazmín, que Sahagún acota con el adjetivo indiano, "jazmjn indiano que ellos llaman vmjsuchitl" (Ap. L. V, f. $345 \mathrm{v}$.), aunque con referencia precisa al omisóchil (n. omixochitl) o lirio americano $^{25}$. Incluso las voces indias, aclimatadas ya a la lengua, recibieron esta especificación, como la batata, que identifica, como no podía ser de otra manera, al camote mexicano: "Otras raýzes buenas de comer que se hazen como nabos debaxo de la tierra, a las quales llaman camotli. Estas son vatatas deSta tierra, cómense cozidas, crudas y aSsadas" (L. XI, f. 279v.).

Las sustancias y materiales también llevaron este distintivo. El copal, que ejercía las mismas funciones que el incienso en las ceremonias religiosas, fue llamado incienso de la tierra o incienso blanco, "eSte betún mézclase con el copal o encienso de la tierra y con la reSina odorífera" (L. X, f. 67v.); "vende también el betún que es como pez y el encienSo blanco y agallas para hazer tinta" (L. X, f. 57v. $)^{26}$, porque, como comenta Sahagún, "no vsauan del incienso aunque lo ay en esta tierra" (Ap. L. II, f. 174v.); el aje (n. axin), también con la adjetivación de color, recibió el nombre de ungüento amarillo de la tierra $\mathrm{o}$, el menos específico, tinta de la tierra, por sus propiedades curativas y tintóreas, que sanaba enfermedades y embellecía el rostro de las mujeres aztecas, "Suélese también vntarse con vngüento amarillo de la tierra, que llaman axí, para tener buen rostro y luziente" (L. X, f. 41r.), "también Se

23 La descripción sahaguntina informa sobre las diferencias entre ambas especies: "ay vnus arboles que en parte parecen a las palmas porque tienen vnas hojas como las palmas, pero no tienen ramas como palmas [...] y hazen vn fructo que parecen dátiles y Son muy dulces y Son muy buenos de comer" (L. XI, f. 343r.) / tx. n. icçotl.

24 Su aparición en los contexos enumerativos de las distintas especies certifica su empleo: "vende también todo lo sigujente, vnos erizos de fruta, vna fruta como nabos, vnas raýzes de árboles que Son como patatas y patatas siluestres y vnas raýzes comestibles llamadas tolcimatl y vnas raýzes que tienen comer de castañas, y piñas fruta y tzapotes amarillos, tzapotes negros de dentro, peruétanos, anonas, mameyes, ciruelas verdes y amarillas, guayauas, mançanjllas de la tierra, cerezas de qualqujer especie" (L. X, f. 60r.).

25 Como 'lirio' recogen esta voz náhuatl, SM; Neves y Santamaría, asimismo, para México.

26 También como adjetivo que acompaña al nahuatlismo, "incienso que se llama copalli blanco" (Ap. L. II, f. 199r.) 
rebueluen eStos poluos con tinta de la tierra, pegado con su pluma, quando el enfermo no tiene calentura" (L. XI, f. 320v.); y la navaja de la tierra fue denominación general del itztetl, instrumento cortante extraído de una especie de piedra de obsidiana, "se ha de tomar vna punta de nauaja de la tierra y punçar la cabeça" (L. X, f. 100r.); "papel, pedernal, nauaja de la tierra" (L. X, f. 40v.), "el official de las nauajas de la tierra sácalas de piedra negra con vn instrumento de palo" (L. X, f. 64r.). Asimismo, el sintagma cristal de la tierra tradujo la voz náhuatl tehuilotl, denominación azteca del 'vidrio', que no ha perdurado, "el criStal deSta tierra Se llama tevilotl, es piedra que se halla en mjnas en las montañas" (L. XI, f. 358v.); y, con una bonita explicación sobre el sentido literal del término se recoge el ámbar de la tierra, que los indios llamaban apoçonalli, "porque el ámbar deSta tierra o eStas piedras ansí llamadas Son semejantes a las campanjtas o enpollas del agua, quando las da el Sol, en Saliendo, que parece que Son amarillas claras, como oro" (L. XI, f. 358v.), ambos como ejemplos de equivalencias léxicas entre ambas lenguas, aunque no siempre estuviera seguro nuestro franciscano de esta identidad referencial, a tenor de sus apreciaciones, cuando comenta a propósito del tlapalteoxihuitl, "creo que Son rubíes deSta tierra" (L. XI, f. 358r.). También las enfermedades indígenas encontraron su equivalente patrimonial, acompañadas del sintagma prepositivo, como el nanahuate (n. nanahuatl), especie de bubas, que Sahagún reconoce como sarna de la tierra, "dase a beber eSta agua a los que tienen Sarna de la tierra que se llama nanavatl" (L. XI, f. 308v.).

Algunas denominaciones tuvieron una especifícación geográfica más concreta, que nos habla del conocimiento de estos hombres del mundo americano, y del tránsito de estos especímenes de la fauna y de la flora entre los distintos lugares de América. Al área del antiguo imperio incaico se ciñen las varias referencias a los patos o ánades del Perú, que el sahaguntino utiliza reiteradamente como elemento comparativo, "otra ave del agua ay que se llama quacoztli [...], es del tamaño de vn pato de los del Perú" (L. XI, f. 190r.); "ay otras aues del agua que se llaman colcanauhtli [...], es del tamaño de los patos del Perú" (L. XI, f. 193r.). Con acotación espacial aparece asimismo, la mata de Chile, "las yeruas comestibles como son las hojas de las matas de Chile, bledos, azederas, mastuerço, poleo y otras muchas yeruas buenas para comer" (L. X, f. 70r./v.), denominación imprecisa de una hierba comestible. Incluso, el huipil, que otros cronistas llaman camisa de la tierra, recibe sistemáticamente la doble determinación, como camisa de las mujeres de esta tierra o como camisa de las mujeres de México, "su officio es tratar en las mantas de los hombres y en las camjSas de las mugeres desta tierra que se llama vipiles, que Son galanas y muy bien labradas" (L. X, f. 47r.), "Son a manera de vipiles que Son camjSas de 
las mugeres de México" (L. X, f. 141r.), con una circunscripción al imperio azteca, y a su uso femenino que destaca la dimensión etnográfica de estos escritos, con un análisis pormenorizado de los elementos culturales indios ${ }^{27}$.

No se agota con esto la lista de procedimientos con los que se nombraron las cosas de América. En ocasiones, fueron las características más sobresalientes de estas plantas y animales las que proporcionaron el elemento diferencial que se fijó como designación lingüística en el habla de los conquistadores y de los colonos. Conocidas son las variadas denominaciones del pavo americano, el guajolote de México, como gallina de la tierra, pero también como gallo de papada, "ay vnas aues en el agua que se llaman atotoli [...], es tamaña como vn gallo de papada" (L. XI, f. 181r.), "gallinas [...] ora sean de la tierra, ora de Castilla, gordas, tiernas, nuevas, pollos y gallos que tienen papada" (L. X, f. 63v.); porque, como explica Sahagún, "Los machos se llaman vexolotl y tienen gran papada y gran pechuga" (L. XI, f. 208v. $)^{28}$. En otras, el parecido parcial con los frutos de Castilla obligaba a introducir, también a partir del mecanismo sintagmático, el rasgo que los oponía, porque poco tenía que ver nuestra castaña con el cacomite (n. cacomitl), raíz tuberculosa que los indígenas comían cocida, salvo en que "tiene Sabor como de castañas" (L. XI, f. 280v.), como afirma reiteradamente Sahagún, y explica esta divergencia con la recurrencia al sintagma castaña de raíz (de hierbas), "los que vendían turrones de chian, caStañas de raýzes de yerua, raýzes como regaliz" (L. VIII, f. 299v.), que evidencia la disparidad de ambos productos; o la piña europea con el ananás, tan diferentes en el fruto, como reconoce Sahagún, cuando especifica la segunda como piña fruta, "vnas raýzes que tienen comer de castañas, y piñas fruta y tzapotes amarillos" (L. X, f. 59v.). Por último, esta semejanza entre las especies, que ya apreciaron los nativos, proporcionó denominaciones que se crean, no tanto como novedad en la lengua de los colonos, como por la traducción de las voces del náhuatl. Un ejemplo de ello es el término perro de agua, que Sahagún identifica con la nutria, con noticias sobre su uso y el contraste léxico entre las variedades, "ay otro anjmal al qual llaman perro del agua porque viue en el agua. Estos son los que nosotros llamamos notrias" (L.

\footnotetext{
27 Una denominación más cercana a la que daba el propio Molina en su Vocabulario, como camisa de india (Alvar 1970 / 1990: 27).

28 Alvar (1970 / 1990: 28), que recuerda la descripción sahaguntina, menciona las denominaciones patrimoniales gallina, gallina de la tierra, gallo o gallo de papada, junto a otras de origen indígena, como la antillana guanaja o la náhuatl guajolote de Fernández de Oviedo, y comenta como algunas de ellas perduran en los distintos países americanos, como gallina de la tierra, en Nuevo Méjico y Colorado, o pavo del país en Yucatán.
} 
XI, f. $171 \mathrm{v}.)^{29}$, y que sustituye en esta obra al indígena aitzcuintli, formado a partir de los lexemas atl 'agua' e itzcuintli 'perro', aunque la construcción ha mantenido cierta vigencia, al menos en México, como nombre genérico de estos mamíferos carniceros ${ }^{30}$.

\section{CONCLUSIÓN}

Sin resultar estrictamente novedoso, la crónica sahaguntina muestra, a partir de los reiterados procesos analógicos y metafóricos, y de los mecanismos lexicogenésicos de la lengua, el proceso de adaptación conceptual y lingüística al mundo americano, atingente a todos los ámbitos del vocabulario, pero más frecuente en el de la flora y la fauna indígenas. No todas estas voces lograron un asentamiento definitivo en nuestro idioma, $y$, probablemente, ni siquiera en el idiolecto de nuestro franciscano, pero la pervivencia de no pocos vocablos, generalmente en convivencia con los términos autóctonos, que se comprueba en las obras lexicográficas americanas, justifican, aún más si cabe, la atención prestada a esta parcela del estudio histórico del léxico, y en el caso de la obra de nuestro fraile, su presentación lexicológica, con la traducción de las voces en ambas lenguas y su comentario descriptivo, resulta un testimonio excepcional de los problemas surgidos ante la nueva cultura, con datos sobre los procedimientos más recurrentes y la constatación de las diferencias que dificultan esta nominación. Asimismo, el análisis de esta crónica, como la de cualquier texto americano, permite obtener, más en el contexto geográfico de México, y en el periodo en el que se inscribe, datos sobre la geografía y la estratigrafía de las voces, con noticias explícitas sobre el asentamiento de los vocablos en el territorio mexicano, y la consecuente dialectalización americana, y la migración de otras, desde distintas zonas del continente.

29 Muy diferente de la equivalencia léxica que solo se documenta en la descripción etnográfica de la especie: "a la nutria llaman aitzcujntli, la qual también anda en el agua" (L. XI, f. 222r.)

30 Según se recoge en los diccionarios generales de americanismos de Morínigo y de Neves (s. v. perro), en este último, como general, y en $S M$, s. v. perro; y mantiene el diccionario académico en su edición actual, también en el avance de la vigésima tercera (s. v. perro), aunque esté ausente de la nómina de voces del $D A A$. 


\title{
REFERENCIAS BIBLIOGRÁFICAS
}

Alvar, Manuel. 1970. Americanismos en la Historia de Bernal Díaz del Castillo. RFE, anejo LXXXIX, nueva edición en 1990. Madrid: Ediciones Cultura Hispánica.

\author{
1972. Juan de Castellanos. Tradición española y realidad americana. Bogotá: \\ Instituto Caro y Cuervo. \\ 1976. Diario del Almirante. Las Palmas: Cabildo Insular de Gran Canaria, 2 vols. \\ 1987. Léxico del mestizaje en Hispanoamérica. Instituto de Cooperación \\ Iberoamericana.
}

1992. Cronistas de Indias. En César Hernández Alonso (coord.). Historia y presente del español de América, pp. 25-60. Valladolid: Junta de Castilla y León, Valladolid.

Asociación de Academias de la Lengua Española. 2010. Diccionario de americanismos $(D A A)$. Perú: Santillana Ediciones Generales.

Bastardín Candón, Teresa. 2011. Migración de léxico regional al español americano. La crónica de fray Bernardino de Sahagún. Boletín de Filología, Tomo XLVI, número 2, pp. 11-34.

Boyd-Bowman, Peter. 1972. Léxico hispanoamericano del siglo XVI. Londres: Támesis Books Limited.

Chuchuy, Claudio et al. (coords.). 2000. Diccionario del español en Argentina (EA). Madrid: Gredos, reimpr. 2003.

Corominas, Joan y José Antonio Pascual. 1980-1991. Diccionario crítico etimológico castellano e hispánico (DCECH). Madrid: Gredos, 6 vols.

Enguita Utrilla, José María. 1979. El fondo léxico patrimonial y la nueva realidad americana. Estudios Paraguayos. Revista de la Universidad Católica Nra. Sra. De la Asunción, VII, 1, pp. 165-175.

1987. Sobre la evolución del fondo léxico patrimonial en el Nuevo Mundo. LEA, IX, pp. 139-51.

1991. Recepción de indigenismos en algunos textos cronísticos del siglo XVI. Actas del III Congreso Internacional del Español de América. Valladolid: Junta de Castilla y León, pp. 199-212.

Frago Gracia, Juan Antonio y Mariano Franco Figueroa. 2003. El español de América. Cádiz: Servicio de Publicaciones de la Universidad de Cádiz.

Frago Gracia, JuAn Antonio. 1995. La lengua de los castellanos-leoneses emigrados a Indias. En César Hérnandez Alonso (coord.). La lengua española y su expansión en la época del Tratado de Tordesillas, pp. 79-97. Salamanca: Junta de Castilla y León.

1999. Historia del español de América. Textos y contextos. Madrid: Gredos.

Franco Figueroa, Mariano. 1991. El fondo léxico patrimonial: americanismos semánticos en documentos de los siglos XVI y XVII. Anuario de Lingüística Hispánica, vol. VII, pp. 219-247.

2001. Meridionalismos en documentos hispanoamericanos: cartas de particulares y la Historia General de Sahagún, y la Crónica y buen gobierno, de Guamán Poma de Ayala. Estudios sobre el español de América. Actas del V Congreso internacional del Español de América. Burgos: Universidad de Burgos, pp. 519-543.

Malaret, Augusto. 1946. Diccionario de americanismos. Buenos Aires: Emecé, $3^{\text {a }}$ ed.

Morínigo, Marcos A. 1953 y 1959. La formación léxica regional hispanoamericana. NRFH, VII, pp. 234-241, y en Programa de Filología Hispánica. Buenos Aires: Editorial Nova, pp. 56-70. 
1964. La penetración de los indigenismos americanos en el español. Presente y futuro de la lengua española. Madrid: Ediciones Cultura Hispánica, pp. 217-226.

1985. Diccionario de americanismos. Barcelona: Muchnik Editores, $2^{\mathrm{a}}$ edición.

Neves, Alfredo. 1975. Diccionario de Americanismos. Madrid: Sopena, $2^{\mathrm{a}}$ edición.

Real Academia de la Lengua Española. Diccionario de la lengua española [en línea]. Madrid: Real Academia de la Lengua Española. Disponible en <http://www.rae.es> [en línea].

1726-1739 / 1980. Diccionario de Autoridades. Madrid: Gredos, edición facsímil, 3. vols.

Rivarola, José Luis. 1990. La formación lingüística de Hispanoamérica. Lima: Pontificia Universidad Católica del Perú.

Sahagún, Fray Bernardino de. 1979. Códice Florentino. Historia general de las cosas de la Nueva España. Manuscrito 218-20 de la Colección Palatina de la Biblioteca mediceaLaurenziana. México (Florencia): Casa editorial Giunti Barbèra, Archivo General de la Nación, edición facsímil, 3 vols.

SANTAMARÍA, Francisco DE. 1942-1943. Diccionario general de americanismos. México, 3 vols. 1983. Diccionario de mejicanismos (SM). México: Editorial Porrúa, $4^{\text {a }}$ edición corregida y aumentada. 